International Journal of Biomedicine | June 2019 - Volume 9, Issue Suppl_1: Abstracts From the Second Russian International Conference "Cryo-electron microscopy 2019: achievements and prospects"

POSTER ABSTRACT PRESENTATIONS

SESSION TITLE: COMPLEX AND EMERGING TECHNIQUES IN STRUCTURAL BIOLOGY

DOI: 10.21103/IJBM.9.Suppl_1.P44

\title{
Abstract P-44: Dimer Structure of Highly Thermostable Archaeal Beta- Galactosidase Revealed by Cryo-EM
}

\author{
Alexander A. Cheblokov ${ }^{1}$, Evgeny B. Pichkur ${ }^{1,2,4}$, Yury V. Kil ${ }^{2}$, \\ Vladimir R. Sergeev ${ }^{2,3}$, Georgy N. Rychkov ${ }^{2,3}$
}

${ }^{1}$ Petersburg Nuclear Physics Institute named by B.P.Konstantinov of National Research Center "Kurchatov Institute", Gatchina, Russia; ${ }^{2}$ National Research Center "Kurchatov Institute", Moscow, Russia; ${ }^{3}$ Peter the Great Saint-Petersburg Polytechnic University, St. Petersburg, Russia; ${ }^{4}$ FSRC "Crystallography and Photonics", Moscow, Russia

Background: Studies on beta-galactosidases (EC3.2.1.23) are of a great value for the optimization of industry production of low-lactose or lactose-free dairy products. A new 975 amino acid $D a-\beta$-gal (proposed to be a GH35 family member) from hyperthermophilic archaeon Desulfurococcus amylolyticus possesses high thermoactivity and thermal stability at temperatures closed to $100^{\circ} \mathrm{C}$.

Methods: Negative staining TEM with $1 \%$ uranyl acetate was performed for sample characterization prior to the cryo-EM studies. Preliminary Cryo-EM dataset was collected using Titan Krios (Thermo-Fisher, USA) equipped with the direct electron detector Falcon II at 300kV accelerating voltage. Movie stacks were preprocessed using Warp. All further processing steps were performed in CryoSPARC and Relion 3.0. Constructed full-atom model of dimer formed by similar mesophilic betagalactosidase from Trichoderma reesei (Tr- $\beta$-gal), PDB entry $3 \mathrm{OGV}$, was refined by molecular dynamics (MD) simulations in explicit water box with periodic boundary conditions in Amber16 (ff14SB, TIP3P, PME, 1 atm, 300K, $100 \mathrm{~ns}$ ).

Results: The Da- $\beta$-gal enzyme, expressed in Escherichia coli cells and purified to homogeneity by column chromatographies, was subjected to negative contrast microscopy and Cryo-EM. Analysis of electron density distributions in collected set of $30 \mathrm{~K}$ appropriately classified individual particle images allowed us to solve the structure of the enzyme at $7 \AA$ resolution. The symmetry of obtained structure has revealed that in utilized buffer conditions the enzyme exists as a dimeric form. MD simulations of $T r-\beta-$ gal hypothetical dimer, have shown stability of the complex along $100 \mathrm{~ns}$ trajectory. As estimated from the model the protomer of $\operatorname{Tr}$ - $\beta$-gal buries about $7.5 \%$ of solvent accessible surface area into dimer interface. 
Conclusion: The preliminary CryoEM data in concert with molecular modeling identifies dimeric quaternary structure of a $111-\mathrm{kDa} D a-\beta$-gal that can be assumed as a structural basis for high temperature stability of the enzyme.

Key Words: beta-galactosidase $\bullet$ protein 3D structure $\bullet$ thermostable enzymes $\bullet$ cryo-EM

Sources of Funding: This work was supported by the Russian Science Foundation under grant 19-7420146. The authors acknowledge the support and the use of resources of the Resource Center for Probe and Electron Microscopy at the NRC "Kurchatov Institute".

International Journal of Biomedicine. 2019;9 Suppl 1: S36-37. doi: 10.21103/JJBM.9.Suppl_1.P44

C2019 International Medical Research and Development Corporation 